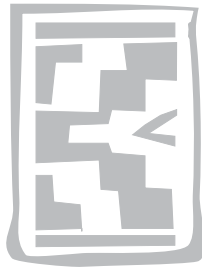

\title{
A potential krimpsiekte vaccine
}

\author{
C.J. BOTHA ${ }^{1 *}$, J.E. CRAFFORD², V.P. BUTLER Jr³, M.N. STOJANOVIC ${ }^{3}$ \\ and L. LABUSCHAGNE 4
}

\begin{abstract}
BOTHA, C.J., CRAFFORD, J.E., BUTLER, V.P. Jr, STOJANOVIC, M.N. \& LABUSCHAGNE, L. 2007. A potential krimpsiekte vaccine. Onderstepoort Journal of Veterinary Research, 74:307-314

Krimpsiekte, a chronic form of cardiac glycoside poisoning, is an important plant-induced intoxication of small stock in South Africa. It is caused by cumulative, neurotoxic bufadienolides, such as cotyledoside. A cotyledoside-bovine serum albumin conjugate was synthesized to immunize animals. The efficacy of the cotyledoside-conjugate in inducing an immunological response was ascertained in rabbits $(n=4)$ and sheep $(n=4)$ by determining cotyledoside antibody titres with an ELISA using cotyledoside-hen ovalbumin as antigen. The formation of anticotyledoside antibodies was induced in both rabbits and sheep following immunization with the cotyledoside-protein conjugate. Protection provided by the vaccine was demonstrated by challenging sheep $(n=4)$ with repeated, daily doses of cotyledoside $(0.015 \mathrm{mg} / \mathrm{kg})$ administered intravenously, commencing 45 days after the initial vaccination. One control animal died on Day 3 of the challenge period and the other was severely affected after administration of the third cotyledoside dose. The immunized ewes $(n=2)$ remained clinically unaffected and the challenge was suspended following six daily injections. Vaccination as a means of preventing krimpsiekte seems to be quite feasible and deserves further investigation.
\end{abstract}

Keywords: Cardiac glycoside, cotyledoside, krimpsiekte, immunization, rabbits, sheep, vaccine

\section{INTRODUCTION}

Poisoning of livestock by cardiac glycoside-containing plants has the greatest economic impact of all plant-associated poisonings in the Republic of South

* Author to whom correspondence is to be directed: E-mail: christo.botha@up.ac.za

1 Department of Paraclinical Sciences, Faculty of Veterinary Science, University of Pretoria, Private Bag X04, Onderstepoort, 0110 South Africa

2 Department of Tropical Diseases, Faculty of Veterinary Science, University of Pretoria, Private Bag X04, Onderstepoort, 0110 South Africa

3 Department of Medicine, College of Physicians and Surgeons, Columbia University, 630 West 168th Street, New York NY 10032 United States of America

4 Toxicology Division, ARC-Onderstepoort Veterinary Institute, Private Bag X05, Onderstepoort, 0110 South Africa

Accepted for publication 26 April 2007-Editor
Africa (Kellerman, Naudé \& Fourie 1996). Collectively they are held responsible for $33 \%$ of all mortalities from plant poisonings of cattle and $10 \%$ of those in small stock. The majority of the cardiac glycoside poisonings in small stock is ascribed to krimpsiekte, arguably the most economically important plant poisoning of small stock in the Little Karoo and southern Great Karoo (Botha 2003; Kellerman, Coetzer, Naudé \& Botha 2005). It is estimated that more than 26000 sheep and goats succumb annually (Kellerman et al. 1996).

Chemically, two major groups of cardiac glycosides, namely the cardenolides and bufadienolides, are recognized. Poisoning by bufadienolide-containing plants surpasses cardenolide-induced poisonings in importance and may be either acute or chronic. Tulp poisoning (induced by various Moraea species) and slangkop poisoning (caused by various Drimia 
species) induce only acute intoxication as these species contain non-cumulative bufadienolides (Kellerman et al. 1996, 2005).

Members of three genera of the Crassulaceae (Cotyledon, Tylecodon and Kalanchoe), colloquially referred to as plakkies, on the other hand, may cause either acute or chronic poisoning. Krimpsiekte, the chronic form of the poisoning, is a neuromuscular affliction of small stock following ingestion of plants that contain atypical cardiac glycosides (such as cotyledoside and tyledoside D), generally referred to as cumulative, neurotoxic bufadienolides. Tylecodon wallichii and Tylecodon ventricosus are probably the most important species of the group of plants causing krimpsiekte (Botha, Van der Lugt, Erasmus, Kellerman, Schultz \& Vleggaar 1997; Botha, Kellerman, Schultz, Erasmus, Vleggaar \& Retief 1998). In this syndrome the cardiac, respiratory and gastrointestinal signs, typical of acute poisoning are diminished and the neuromuscular signs increase. Small stock tire easily, lag behind the flock and frequently lie down. Often they assume a characteristic stance, with the back arched, limbs tucked in under the body and head down, sometimes trembling. The animals may be recumbent for long periods.

Of particular concern is evidence that secondary poisoning of humans can occur if meat or edible tissues obtained from carcasses of animals that have died of krimpsiekte are consumed (Kellerman et al. 2005). This is a real danger, as rural people with meagre means and financial constraints, commonly consume animals that die in the veld.

Immunoprophylaxis against plant- and fungal poisonings is currently receiving some attention; for instance, Australian researchers are evaluating immunization as a means of preventing the mycotoxicosis lupinosis in sheep (Than, Anderton, Cockrum, Payne, Stewart \& Edgar 1994). Most phytotoxins are not immunogenic due to their small molecular size and, in order to induce immunity following parenteral administration to an animal, must first be coupled to carrier proteins (Silbart, Rasmussen \& Oliver 1997). Various attempts at vaccinating stock against plant toxins have been unsuccessful for a number of reasons. One of these is that the immunological response is overwhelmed due to the high toxin concentrations, which occur in acute toxic exposures (Edgar 1994). Krimpsiekte, on the other hand, is a chronic manifestation that can be induced by repeated exposures to small doses of the cumulative bufadienolide; a vaccine, therefore, could conceivably prevent this intoxication. If a prophylactic vaccine could be produced the incidence of, and mortalities caused by, krimpsiekte could be curtailed.

Technically the development of a vaccine to prevent krimpsiekte is quite feasible (Butler \& Chen 1967; Schmidt \& Butler 1971). Antibodies have been raised against digoxin, a cardenolide cardiac glycoside. Sheep and rabbits immunized by repetitive administration of digoxin-protein conjugates developed high serum titres of antidigoxin antibodies of high specificity and affinity (Schmidt \& Butler 1971; Butler, Smith, Schmidt \& Haber 1977b). Immunized rabbits were protected from the toxic effects of a lethal dose of digoxin (Butler et al. 1977b). However, antibody binding of digoxin interferes with the renal excretion of digoxin, thus greatly prolonging the half-life of this compound (Schmidt, Kaufman \& Butler 1974; Butler, Schmidt, Smith, Haber, Raynor \& Demartini 1977a; Butler et al. 1977b).

The first objective of this project was to synthesize a cotyledoside-protein conjugate with which to immunize animals. The second aim was to evaluate the efficacy of the conjugate in inducing an immunological response in rabbits and sheep by determining cotyledoside antibody titres in an ELISA. The third objective was to demonstrate the efficacy of the vaccine (conjugate) by challenging sheep with the neurotoxic, cumulative bufadienolide, cotyledoside.

\section{MATERIALS AND METHODS}

\section{Preparation of cotyledoside-protein conjugates}

Cotyledoside has previously been extracted and purified from T. wallichii (Botha et al. 1997). Cotyledoside-protein conjugates were prepared by a mixed anhydride procedure (Erlanger, Borek, Beiser \& Lieberman 1957). Cotyledoside (1 mg) was heated in a closed container (oil bath) at $100^{\circ} \mathrm{C}$ in pyridine $(200 \mu \ell)$ in the presence of succinic anhydride $(1 \mathrm{mg})$ and a catalytic amount of dimethylaminopyridine for $8 \mathrm{~h}$. During this time, the reaction mixture turned distinctly brown. The reaction was followed by removal of aliquots $(2 \mu \ell)$ with a micropipette, quenching them in methanol $(100 \mu \ell)$ and confirming by mass spectra (Shimadzu LCMS 2010A) the disappearance of the starting material, with the concomitant production of succinylated cotyledoside. No further characterization was performed on this material. The reaction was stopped by the removal of heat and addition of ethyl acetate. Solvent was removed by a stream of nitrogen and the residue was partitioned between $0.01 \mathrm{M} \mathrm{HCl}$ solution and ethyl acetate $(1 \mathrm{~m} \ell$ each). The organic layer was 
dried over magnesium sulphate. After evaporation of solvent under vacuum, the residue was dissolved in tetrahydrofuran (THF, $100 \mu \ell$ ). The acid was transformed into the $\mathrm{N}$-hydroxysuccinimide (NHS) ester by the addition of dicyclohexyl carbodiimide, (DCC, $1 \mathrm{mg})$ and NHS (1 mg). Within $1 \mathrm{~h}$, the precipitate was filtered through a wool pad and added directly to a protein solution ( $5 \mathrm{mg}$ of either bovine serum albumin (BSA) or hen ovalbumin (OVA) in $2 \mathrm{~m} \ell$ water) and the $\mathrm{pH}$ was adjusted to 8 by adding $\mathrm{NaHCO}_{3}$. The reaction mixture was stirred overnight and then filtered, THF was evaporated under vacuum and excess small molecules were removed by gel filtration (PD10 column, Pharmacia). Accumulated protein fractions had characteristic cotyledoside UV peaks $(\sim 300 \mathrm{~nm})$, indicating attachment between 4-8 cotyledosides per protein (Fig. 1).

\section{Cotyledoside ELISA}

Maxisorb 96-well plates (NUNC) were coated with $50 \mu \ell$ antigen (OVA-cotyledoside conjugate) at a dilution of $10 \mu \mathrm{g} / \mathrm{m} \ell$ in coating buffer $(0.1 \mathrm{M}$ carbonatebicarbonate buffer, $\mathrm{pH}$ 9.6) in alternate rows. The remaining rows were coated with OVA instead of antigen at the same concentration. The plates were sealed and incubated overnight at $4^{\circ} \mathrm{C}$. Plates were washed five times with PBS supplemented with $0.05 \%$ Tween 20 and blotted dry (washing), then blocked with $100 \mu \ell$ PBS supplemented with $5 \%$ skimmed milk powder per well and incubated for $1 \mathrm{~h}$ at room temperature followed by washing. Sera were loaded in duplicate rows (e.g. row A coated with antigen and row $B$ coated with OVA) and a two-fold dilution series ( $50 \mu \ell$ per well) (using blocking buffer and starting at 1:500 for rabbit sera and 1:200 for sheep sera) was made across the plate followed by

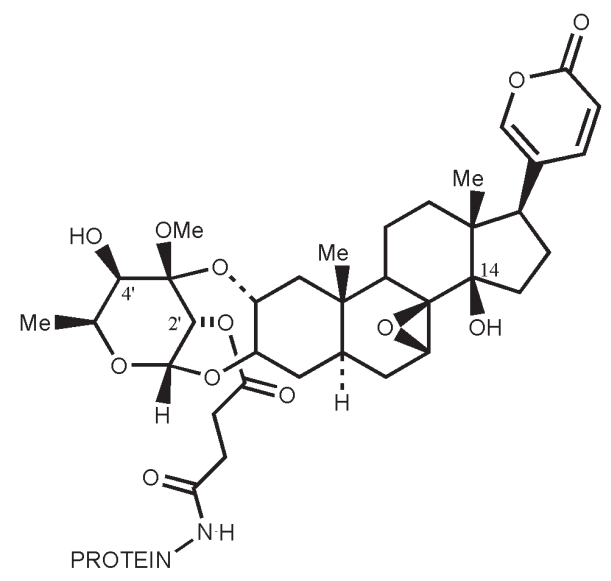

FIG. 1 The cotyledoside-protein conjugate. Note that the conjugation could also possibly occur at position C-4', or at both positions $\mathrm{C}-\mathrm{2}^{\prime}$ and $\mathrm{C}-\mathrm{4}^{\prime}$ incubation and washing. When testing rabbit sera $50 \mu \ell$ biotinylated goat anti-rabbit serum at a dilution of 1:1000 was added to all wells and incubated. After washing, $50 \mu \ell$ strepavidin horseradish-peroxidase (HRPO) conjugate at a dilution of 1:1000 was added to all wells. For testing sheep sera rabbit antisheep HRPO conjugate was used at 1:1000. Following further incubation and washing, $50 \mu \ell$ substrate (ortho-phenylenediamine at a concentration of 0.04 $\mathrm{mg} / \mathrm{m} \ell$ and containing $0.05 \% \mathrm{H}_{2} \mathrm{O}_{2} 30 \% \mathrm{v} / \mathrm{v}$ ) was added to all wells and the plates incubated for 10 min in the dark after which $50 \mu \ell$ stop solution ( $1 \mathrm{~N}$ $\mathrm{H}_{2} \mathrm{SO}_{4}$ ) was added to all wells. The plates were read in a BioTek EL808 plate reader at $450 \mathrm{~nm}$ and results recorded. The net optical density was calculated by subtracting the value obtained for the OVAcoated well from the corresponding antigen-coated well.

\section{Rabbit immunization}

New Zealand White rabbits $(n=4)$ were housed at the University of Pretoria Biomedical Research Centre. They were kept individually in rabbit cages, had free access to water and were fed a commercial ration. For immunization, an emulsion was prepared by adding the antigenic hapten-carrier conjugate $(2 \mathrm{mg} / \mathrm{m} \ell)$ or pure BSA (Sigma) dissolved in normal saline $(2 \mathrm{mg} / \mathrm{m} \ell)$ to an adjuvant, at first to complete and later to incomplete Freund's (Sigma) (Table 1). Two rabbits (a male and a female) were immunized with BSA-cotyledoside conjugate and the other two control rabbits (a male and a female) were immunized with similar volumes of BSA on Days 0,21 and 49 (Table 1). A large area on the back of each rabbit was shaved and they were each immunized by intradermal administration of the in-

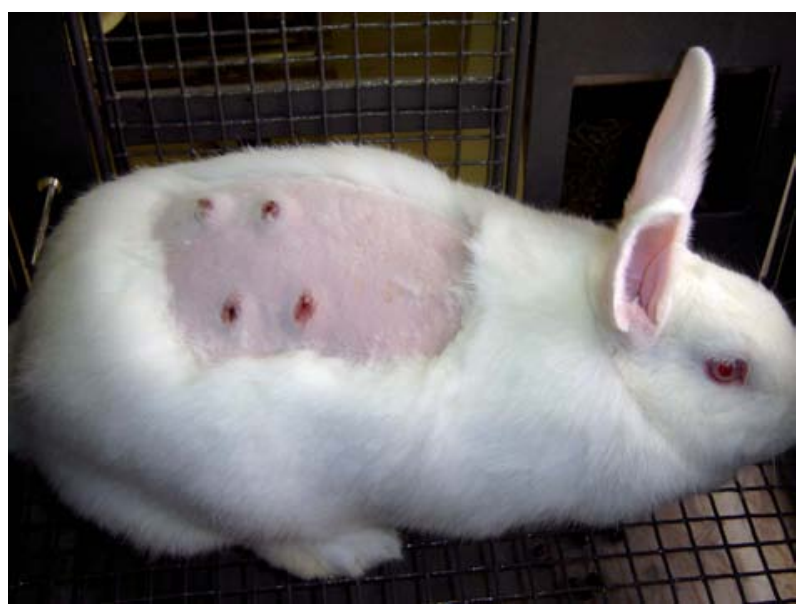

FIG. 2 Rabbit exhibiting granulomatous skin lesions at the injection sites 


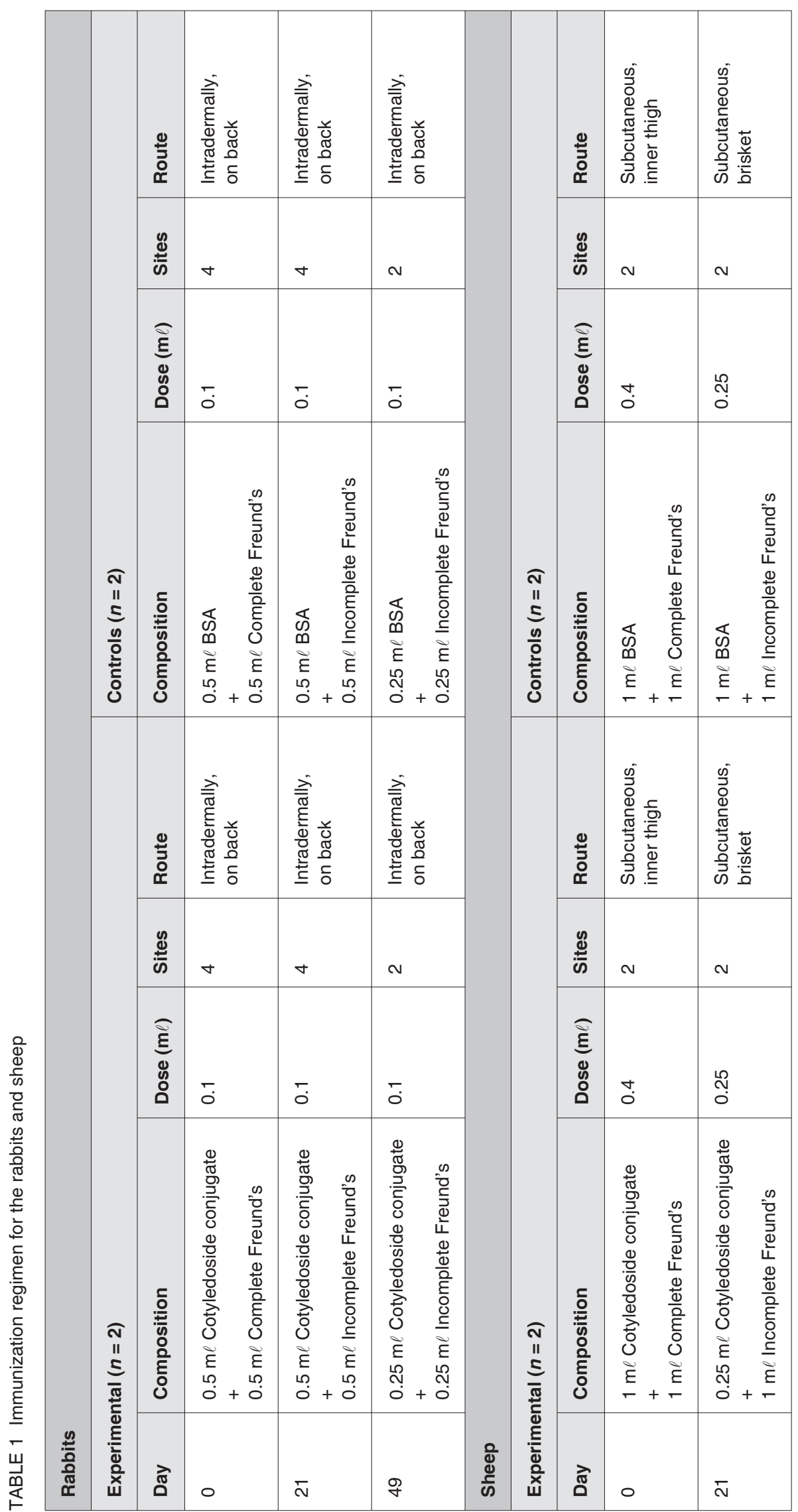


oculum at four sites on the back (Fig. 2). On Days 21 and 49 the rabbits were weighed and bled from an ear vein $(2 \mathrm{~m} \ell)$ to determine antibody titres using the OVA-cotyledoside in an ELISA. On Day 61 the rabbits were anaesthetized (with a xylazine/ketamine combination [Xylavet 2\%, Intervet SA (Pty) Ltd and Anaket, Bayer Animal Health Division]) and exsanguinated by inserting a hypodermic needle into the heart. All the blood was collected for serology.

\section{Sheep immunization}

Four Mutton Merino ewes, weighing $38.5-44.5 \mathrm{~kg}$, were housed in a pen with a concrete floor at the Toxicology Biolab, Onderstepoort Veterinary Institute (OVI). They had free access to water and were fed the OVI standard concentrate and Eragrostis hay. The sheep were randomly allocated to two groups. Two of the sheep were initially immunized by subcutaneous inoculation of an emulsion containing equal volumes of BSA-cotyledoside plus complete Freund's adjuvant. The other two animals served as controls and were vaccinated with the same volumes of BSA dissolved in saline and complete Freund's adjuvant (Table 1). A booster vaccination containing cotyledoside conjugate plus incomplete Freund's adjuvant was prepared and inoculated 3 weeks later (Table 1). The serum antibody titres of all four sheep were determined by ELISA before vaccination, 3 weeks after the initial vaccination and 3 weeks after the booster vaccination (Day 42).

\section{Cotyledoside challenge in sheep}

Commencing 45 days after the initial vaccination, all four sheep were challenged with daily injections of cotyledoside. Cotyledoside (20 mg), previously extracted and purified from $T$. wallichii (Botha et al. 1997), was dissolved in $2 \mathrm{~m} \ell$ ethanol and $78 \mathrm{~m} \ell$ normal saline $(0.025 \% \mathrm{~m} / \mathrm{v})$. Each animal received daily intravenous injections of $0.015 \mathrm{mg} / \mathrm{kg}$ cotyledoside (Botha et al. 1997).

During the challenge study clinical examinations were performed daily. In order to avoid any bias the senior author (CJB), who interpreted the clinical presentations, was blinded to the immunization status of the sheep.

\section{RESULTS}

\section{Rabbit immunization}

All the rabbits developed an injection site reaction, noticeable as erythema and oedema, followed by granulomatous lesions, superficial skin necrosis and scab formation (Fig. 2). High anticotyledoside antibody titres were detected on termination of the experiment (on Day 61) in the two rabbits that were immunized with the BSA-cotyledoside conjugate (Fig. 3). As antibodies were raised in rabbits following immunization, it was decided to continue with the project in sheep, two being subsequently vaccinated.

\section{Sheep immunization}

All four sheep developed slight to severe injectionsite reactions, but this did not hamper their habitus. Anticotyledoside antibodies were raised in the two sheep following immunization with the cotyledosideprotein conjugate (Fig. 4).

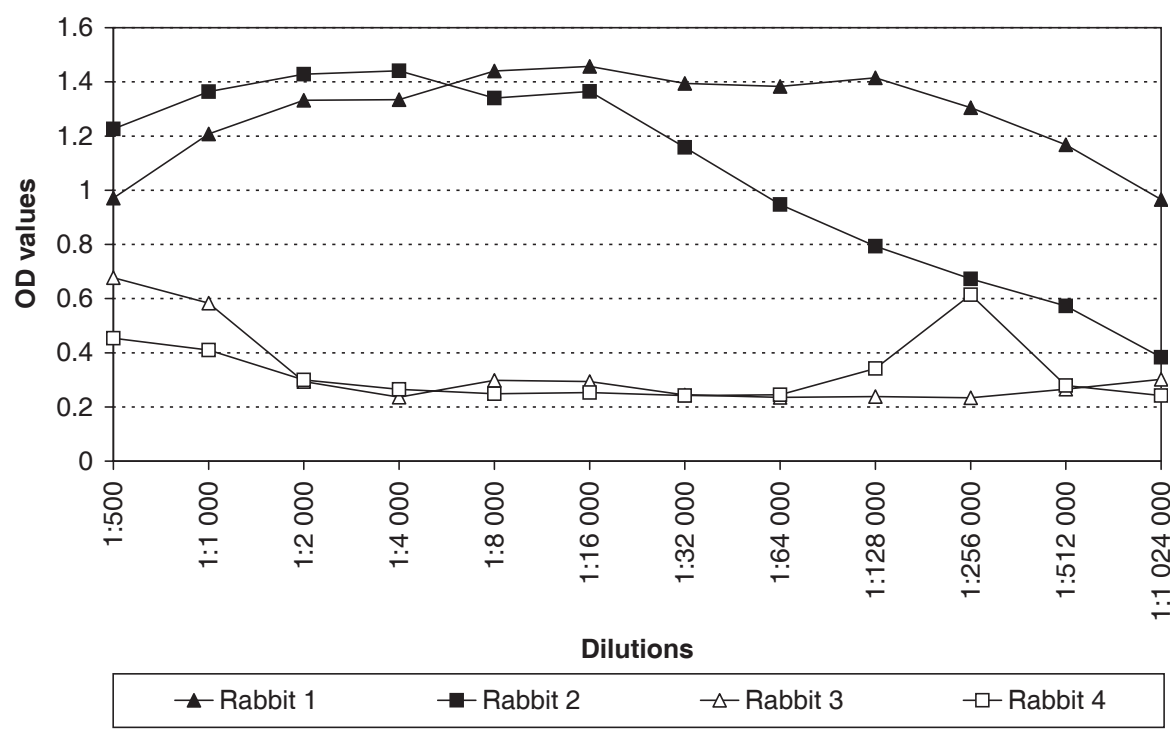

FIG. 3

Optical density values (490 nm) obtained in a cotyledoside ELISA for the rabbit sera on Day 61 . Rabbits 3 and 4 were controls 


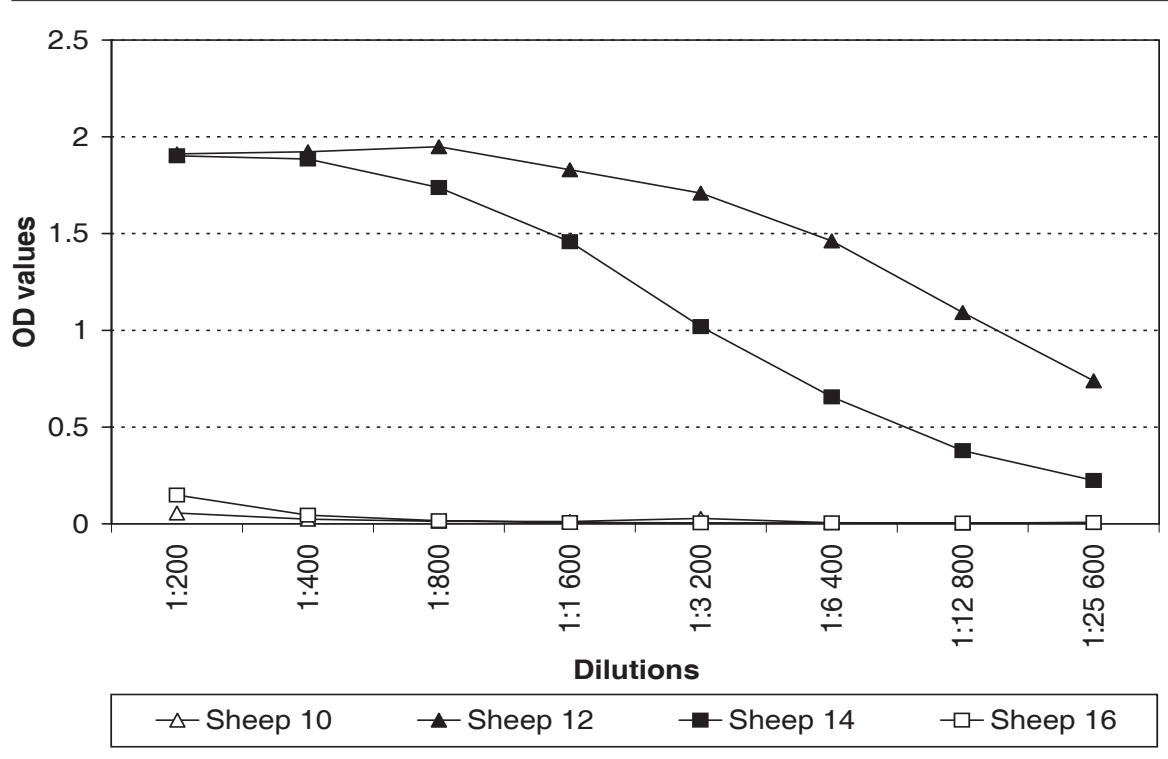

FIG. 4

Optical density values (490 nm) obtained in a cotyledoside ELISA for the sheep sera on Day 42. Sheep 10 and 16 were controls

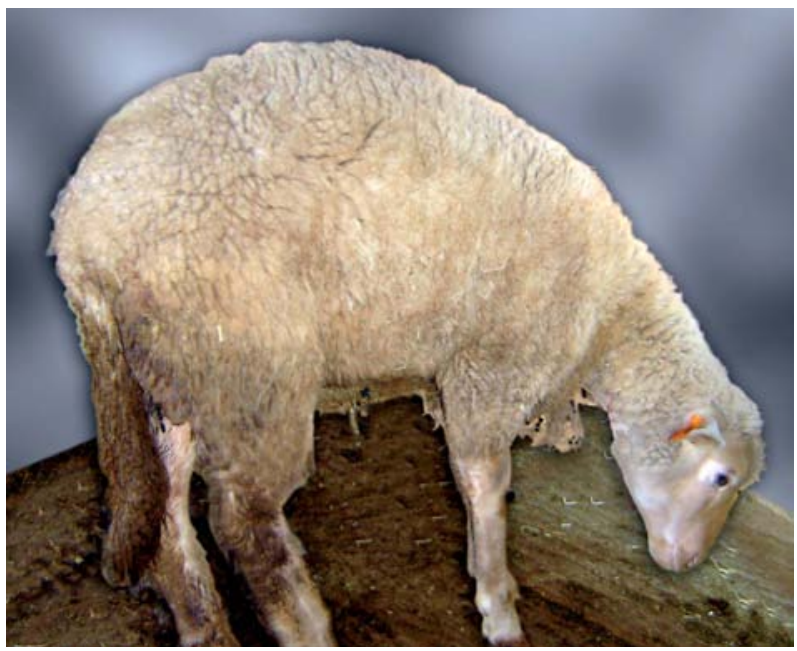

FIG. 5 Affected sheep (Sheep 16) assuming the krimpsiekte posture

\section{Cotyledoside challenge in sheep}

The cotyledoside challenge was suspended when clinical signs reminiscent of krimpsiekte were elicited. One of the sheep developed jerky, forced abdominal respiration and made grunting noises shortly after the third injection administered in the morning. She became paretic during the afternoon and tended to assume the krimpsiekte posture the next day (Fig. 5). Due to ethical considerations no further cotyledoside was administered to this ewe. It transpired that this was a control animal.

Another ewe had rumen stasis on the $4^{\text {th }}$ day, but did not exhibit signs of paresis and was standing. This animal was not judged to be severely affected and it was decided to administer another dose of cotyledoside to this animal. However, shortly thereafter, her condition rapidly deteriorated and she developed severe respiratory distress and died during the afternoon. It was then revealed that this was also a control sheep.

As no sign of toxicity was observed in the other two immunized ewes the daily cotyledoside administrations were continued for a further 2 days (in total six daily injections). These sheep remained clinically unaffected and the challenge was stopped as they had received cotyledoside for a longer period (1.52.0 times) than the control animals. These two sheep remained clinically unaffected for 6 months.

\section{DISCUSSION}

This preliminary trial verified that the cotyledosideprotein conjugate used did indeed induce the formation of high anticotyledoside antibody titres in rabbits and sheep.

In addition, the prophylactic potential of this "monovalent vaccine" was demonstrated in the limited challenge study in the sheep. The cotyledoside conjugate induced an immunological response, which prevented cotyledoside-induced krimpsiekte in the two sheep immunized with it. The death of one of the control animals was unexpected as none of the sheep in previous experiments died after receiving similar amounts of cotyledoside intravenously, and although affected, following cessation of the administration of the toxin they all recovered spontaneously or after administration of strained ruminal content (Botha et al. 1997; Botha, Rundberget, Swan, Mülders, Flåøyen 2003). 
As the duration of challenge of the two cotyledoside-conjugate immunized sheep was 1.5-2.0 times longer than that of the other two sheep, the daily cotyledoside administration to them was stopped to prevent saturation of the induced antibodies with cotyledoside. This could eventually have resulted in excess circulating cotyledoside and, as consequence, death. It can be concluded that immunization of sheep and goats can most probably be utilized as a means of preventing krimpsiekte.

One possible drawback is that animals with high antibody titres might be able to accumulate toxic amounts of cotyledoside, which could be released when the antibody-toxin complex is degraded (Butler et al. 1977a and b). However, the cotyledosideimmunized sheep remained asymptomatic for 6 months after the challenge period, which provides some evidence that release of cotyledoside from degrading antibodies will not result in recrudescence of toxicity.

Another disadvantage of krimpsiekte immunoprophylaxis is the perceived interference in the elimination of cotyledoside and its subsequent retention, which would greatly increase the total body burden of cotyledoside. Thus, carcasses of animals protected by immunization might not be suitable for human consumption (Edgar 1994; Silbart et al. 1997; Botha 2003). However, secondary poisoning from consumption of immunized animals that have consumed $T$. wallichii may or may not constitute a problem. Conceivably, such protected animals could dispose of antibody-bound glycoside by metabolic degradation or conjugation, e.g. to sulphates and glucuronides, which could be excreted in bile.

Another question that needs to be addressed is the degree of cross-reactivity between different krimpsiekte-inducing cumulative bufadienolides. In preliminary trials, similar vaccines produced from cardenolides (digoxin, ouabain) and non-cumulative bufadienolides (proscillaridin) were unable to protect small stock from acute poisoning induced by dosing 4-5 g/kg milled, fresh $T$. wallichii leaves, ostensibly through lack of cross-immunity (J.P.J. Joubert, unpublished data 1985). This lack of crossimmunity can possibly be circumvented by preparing a polyvalent vaccine consisting of the major bufadienolides.

The above drawbacks, however, should not place any constraints on continuing this preliminary investigation. In addition, the antibodies produced could conceivably be used diagnostically in an immunoassay to detect exposure of sheep (live or slaughtered) to cotyledoside and related bufadienolides.

\section{ACKNOWLEDGEMENTS}

Authors M.N. Stojanovic and V.P. Butler were supported by the National Science Foundation, USA, grants CCF-0621600 and CHE-0533096. M.N. Stojanovic is a Leukemia and Lymphoma Society Scholar.

\section{REFERENCES}

BOTHA, C.J., VAN DER LUGT, J.J., ERASMUS, G.L., KELLERMAN, T.S., SCHULTZ, R.A. \& VLEGGAAR, R. 1997. Krimpsiekte, associated with thalamic lesions, induced by the neurotoxic cardiac glycoside, cotyledoside, isolated from Tylecodon wallichii (Harv.) Toelken subsp. wallichii. Onderstepoort Journal of Veterinary Research, 64:123-128.

BOTHA, C.J., KELLERMAN, T.S., SCHULTZ, R.A., ERASMUS, G.L., VLEGGAAR, R. \& RETIEF, E. 1998. Krimpsiekte in a sheep following a single dose of Tylecodon ventricosus (Burm.f.) Toelken and the isolation of tyledoside D from this plant species. Onderstepoort Journal of Veterinary Research, 65:17-23.

BOTHA, C.J., RUNDBERGET, T., SWAN, G.E., MÜLDERS, M.S.G. \& FLÅØYEN, A. 2003. Toxicokinetics of cotyledoside following intravenous administered to sheep. Journal of the South African Veterinary Association, 74:7-10.

BOTHA, C.J. 2003. Krimpsiekte, a paretic/paralytic syndrome of small stock in South Africa. Ph.D. thesis, Norwegian School of Veterinary Science, Oslo.

BUTLER, V.P. \& CHEN, J.P. 1967. Digoxin specific antibodies. Proceedings of the National Academy of Sciences, 57:71-78.

BUTLER, V.P., SCHMIDT, D.H., SMITH, T.W., HABER, E., RAYNOR, B.D. \& DEMARTINI, P. 1977a. The effects of sheep digoxin-specific antibodies and their Fab fragments on digoxin pharmacokinetics in dogs. The Journal of Clinical Investigation, 59:345-359.

BUTLER, V.P., SMITH, T.W., SCHMIDT, D.H. \& HABER, E. $1977 \mathrm{~b}$. Immunological reversal of the effects of digoxin. Federation Proceedings, 36:2235-2241.

EDGAR, J.A. 1994. Vaccination against poisoning diseases, in Plant-associated toxins, agricultural, phytochemical and ecological aspects, edited by S.M. Colegate \& P.R. Dorling. Wallingford: $C A B$ International.

ERLANGER, B.F., BOREK, F., BEISER, S.M. \& LIEBERMAN, S. 1957. Steroid-protein conjugates. I. Preparation and characterization of conjugates of bovine serum albumin with testosterone and with cortisone. Journal of Biological Chemistry, 228:713-727.

KELLERMAN, T.S., NAUDÉ, T.W. \& FOURIE, N. 1996. The distribution, diagnosis and estimated economic impact of plant poisonings and mycotoxicoses in South Africa. Onderstepoort Journal of Veterinary Research, 63:65-90.

KELLERMAN, T.S., COETZER, J.A.W., NAUDÉ, T.W. \& BOTHA, C.J. 2005. Plant poisonings and mycotoxicoses of livestock in Southern Africa, $2^{\text {nd }}$ ed. Cape Town: Oxford University Press.

SCHMIDT, D.H. \& BUTLER, V.P. 1971 Immunological protection against digoxin toxicity. Journal of Clinical Investigation, 50: 866-871.

SCHMIDT, D.H., KAUFMAN, B.M. \& BUTLER, V.P. 1974 Persistence of hapten-antibody complexes in the circulation of immunized animals after a single intravenous injection of hapten. Journal of Experimental Medicine, 139:278-294. 
SILBART, L.K., RASMUSSEN, M.V. \& OLIVER, A.R. 1997. Immunoprophylactic intervention in chemical toxicity and carcinogenicity. Veterinary and Human Toxicology, 39:37-43.

THAN, K.A., ANDERTON, N., COCKRUM, P.A., PAYNE, A.L. STEWART, P.L. \& EDGAR, J.A. 1994. Lupinosis vaccine:
Positive relationship between anti-phomopsin IgG concentration and protection in Victorian field trials, in Plant-associated toxins, agricultural, phytochemical and ecological aspects, edited by S.M. Colegate \& P.R. Dorling, Wallingford: $\mathrm{CAB}$ International. 